\title{
A Family of Interesting Exact Solutions of the Sine-Gordon Equation *
}

\author{
HUANG De-Bin(黄德斌), LIU Zeng-Rong(刘曾荣), WANG Li-Lian(王立联) \\ LNM, Institute of Mechanics, Chinese Academy of Sciences, Beijing 100080 \\ Mathematical Department, Shanghai University, Shanghai 201800
}

(Received 4 May 1999)

\begin{abstract}
By using AKNS [Phys. Rev. Lett. 31 (1973) 125] system and introducing the wave function, a family of interesting exact solutions of the sine-Gordon equation are constructed. These solutions seem to be some soliton, kink, and anti-kink ones respectively for the different choice of the spectrum, whereas due to the interaction between two traveling-waves they have some properties different from usual soliton, kink, and anti-kink solutions.
\end{abstract}

PACS: $02.30 . J r, 02.30 . H q$

During the last three decades an exciting and active area of research has been devoted to constructing exact solutions for a wide class of nonlinear partial differential equations. Indeed, to find exact solutions plays an important role in the research of the nonlinear science. ${ }^{1}$ In particular, solving the sine-Gordon equation

$$
\frac{\partial^{2} u}{\partial T^{2}}-\frac{\partial^{2} u}{\partial X^{2}}+\sin u=0,
$$

which arises in many branches of mathematical physics, has led to an extensive research ${ }^{2,3}$ and its some kink, anti-kink, breather solutions were found in the inverse-scattering method. Furthermore, from these exact solutions of the integrable sine-Gordon equation, the dynamics and solutions of the perturbed sine-Gordon equation were investigated. ${ }^{4-8}$ Especially, the pattern competition in the damped and forced periodically sine-Gordon equation was observed. This implies that the breather solutions are important in the pattern competition. So it is significant to construct new soliton (or kink, anti-kink) solutions of the sine-Gordon equation.

In this letter, instead of the inverse-scattering method, we consider the sine-Gordon equation by using a similar method to generate exact solutions from stationary solutions in Ref. 9 and introducing the wave function ${ }^{10}$ which is solution of the associated AKNS ${ }^{11}$ (Ablowitz, Kaup, Newell and Segur) system. Firstly we construct directly a family of traveling-wave solutions of the sine-Gordon equation from the constant solutions, which are coincident with those obtained in Ref. 11. Secondly, by this family of traveling-wave solutions, we find a family of interesting new exact solutions of the sine-Gordon equation. Finally, by taking limitations in the spatial direction we find this family of new solutions are like kink, anti-kink and soliton solutions respectively for the different choice of the related parameter (actually this parameter just is the spectrum of the sine-Gordon equation). However, these solutions have some properties different from usual soliton, kink, and anti-kink solutions within the local region of space. Actually, from the explicit expression of this family of new solutions, we find that they contain two traveling-waves with different speeds. By stimulating numerically these solutions, we find that there exist various oscillations and singularities within the local region of space, and we believe that the occurrence of these phenomena just results from the interaction between two traveling-waves.

It is known that eigenvalue of the general AKNS system are defined in the form:

$$
\Phi_{x}=M \Phi, \quad \Phi_{t}=N \Phi,
$$

where $\Phi=\left(\begin{array}{l}\varphi_{1} \\ \varphi_{2}\end{array}\right), M$ and $N$ are two $2 \times 2$ matrices:

$$
M=\left(\begin{array}{cc}
\eta & q \\
r & -\eta
\end{array}\right), \quad N=\left(\begin{array}{cc}
A & B \\
C & -A
\end{array}\right),
$$

where $\eta$ is a real parameter. In components, Eq. (2) may be rewritten as

$$
\begin{aligned}
& \varphi_{1 x}=\eta \varphi_{1}+q \varphi_{2}, \\
& \varphi_{2 x}=r \varphi_{1}-\eta \varphi_{2}, \\
& \varphi_{1 t}=A \varphi_{1}+B \varphi_{2}, \\
& \varphi_{2 t}=C \varphi_{1}-A \varphi_{2} .
\end{aligned}
$$

From the integrable condition $\Phi_{x t}=\Phi_{t x}$, we have

$$
\begin{gathered}
-A_{x}+q C-r B=0, \\
q_{t}-B_{x}-2 q A+2 \eta B=0, \\
r_{t}-C_{x}-2 \eta C+2 r A=0,
\end{gathered}
$$

which are equivalent to the desired nonlinear evolution equations by the suitable choice of $A, B$ and $C$.

By introducing a function known as the wave function ${ }^{10}$

$$
\Gamma=\frac{\varphi_{1}}{\varphi_{2}}
$$

the Eq. (2) is reduced to the Ricaati equations:

$$
\begin{aligned}
& \frac{\partial \Gamma}{\partial x}=2 \eta \Gamma+q-r \Gamma^{2} \\
& \frac{\partial \Gamma}{\partial t}=B+2 A \Gamma-C \Gamma^{2}
\end{aligned}
$$



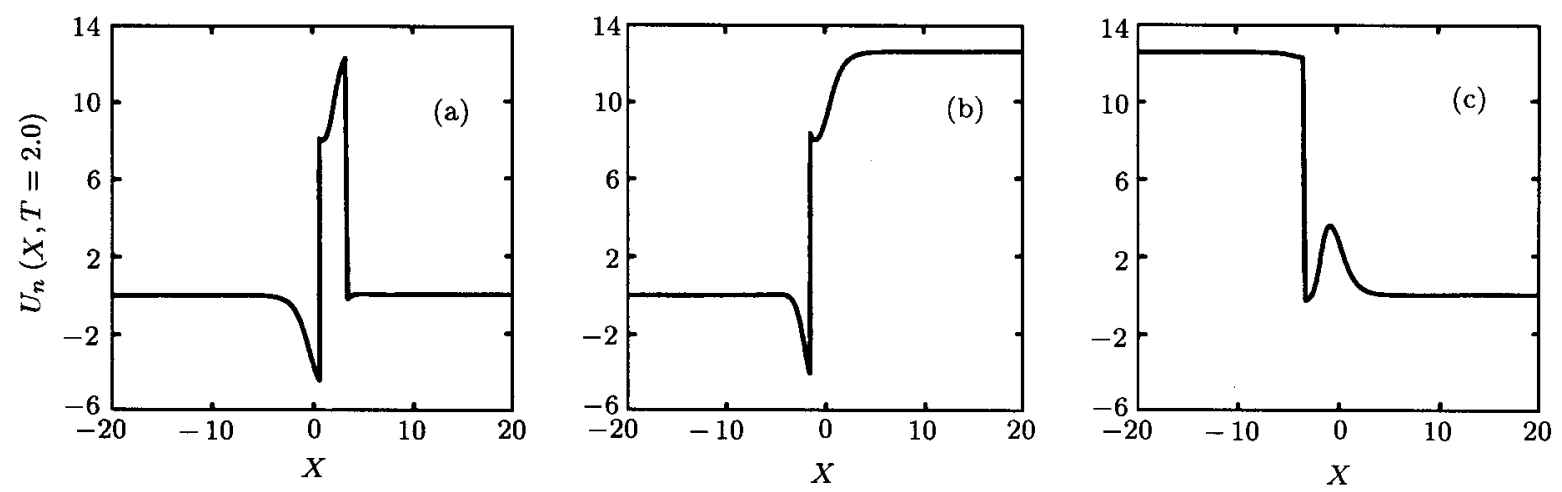

Fig. 1. Profile of the solution with $n=0, c=0, c_{0}=1, T=2.0$ for (a) $\eta=0.25$, (b) $\eta=1$, (c) $\eta=-1$.

Construct a transformation $\Pi:(\Gamma, q) \rightarrow(\widetilde{\Gamma}, \widetilde{q})$

$$
\Gamma \rightarrow \widetilde{\Gamma}, \quad \tilde{q}=q+f(\Gamma, \eta)
$$

where $f$ is a function. If Eq. (9) is invariant under the transformation $\Pi$, i.e. $(\widetilde{\Gamma}, \widetilde{q})$ satisfies Eq. $(9)$, then $\widetilde{q}$ still is a solution of the desired nonlinear equation.

For the sine-Gordon equation (1), by the transformation

$$
x=\frac{1}{2}(X+T), \quad t=\frac{1}{2}(X-T),
$$

Eq. (1) becomes

$$
u_{x t}=\sin u \text {. }
$$

Its corresponding AKNS system is

$$
r=-q=\frac{1}{2} u_{x}, A=\frac{1}{4 \eta} \cos u, B=C=\frac{1}{4 \eta} \sin u .
$$

Actually, substituting Eq. (14) into Eq. (6) gives Eq. (13). In this case, Eq. (9) becomes

$$
\frac{\partial \Gamma}{\partial x}=2 \eta \Gamma+q+q \Gamma^{2} .
$$

Introduce the following transformation

$$
\begin{aligned}
& \widetilde{\Gamma}=\frac{1}{\Gamma}, \\
& \widetilde{q}=q-2 \frac{\partial}{\partial x} \arctan \Gamma .
\end{aligned}
$$

It is obvious that $\tilde{\Gamma}$ together with $\tilde{q}$ satisfies Eq. (15). Therefore, integrating Eq. (17) we obtain the relation between the old and new solutions for the sine-Gordon equation (13)

$$
\widetilde{u}=u+4 \arctan \Gamma
$$

where $\Gamma=\varphi_{1} / \varphi_{2}$. So from a known solution, a new solution for the sine-Gordon equation can be found by Eq. (18).

Let the constant solutions of Eq. (13) be

$$
u_{n}=n \pi, n=0, \pm 1, \pm 2, \cdots,
$$

substituting Eq. (19) into the corresponding AKNS system of the sine-Gordon equation, we get easily

$$
\begin{aligned}
& \varphi_{1}=c_{1} \exp \left(\eta x+\frac{1}{4 \eta}(-1)^{n} t\right), \\
& \varphi_{2}=c_{2} \exp \left(-\eta x-\frac{1}{4 \eta}(-1)^{n} t\right),
\end{aligned}
$$

where $c_{1}$ and $c_{2}$ are the positive constants. So

$$
\Gamma=\frac{\varphi_{1}}{\varphi_{2}}=c_{0} \exp \left(2 \eta x+\frac{1}{2 \eta}(-1)^{n} t\right), c_{0}=\frac{c_{1}}{c_{2}}>0 .
$$

Substituting Eqs. (19) and (21) into Eq. (18), gives

$$
\begin{aligned}
& \tilde{u}=n \pi+4 \arctan \left[c_{0} \exp \left(2 \eta \rho_{n}\right)\right], \\
& \rho_{n}=x-k_{n} t, \quad k_{n}=\frac{(-1)^{n-1}}{4 \eta^{2}}, \quad n=0, \pm 1, \cdots,
\end{aligned}
$$

which are a family of the traveling-wave solutions of the sine-Gordon equation. Furthermore, by using the transformation (12), we find out that the solutions (22) contain the well-known kinks as same as those traveling-wave solutions in Ref. 11.

Next, we want to find a family of new solutions by the above traveling-wave solutions (22). In this case, we suppose that the elements $q$ and $r$ of the matrix $M$ are in the form:

$$
q=q(\rho), \quad r=r(\rho),
$$

where $\rho=x-k t, k$ is a constant. Then by Eqs. (5)-(7), $A, B$ and $C$ read the same form:

$$
A=A(\rho), B=B(\rho), C=C(\rho),
$$

In order to obtain $\varphi_{1}$ and $\varphi_{2}$, from Eq. (4), we have

$$
\begin{aligned}
& \varphi_{1}=\frac{1}{r}\left(\varphi_{2 x}+\eta \varphi_{2}\right), \\
& C \varphi_{2 x}-r \varphi_{2 t}=\frac{1}{2}\left(C_{x}-r_{t}\right) \varphi_{2} .
\end{aligned}
$$

This first-order linear partial differential equation involving $\varphi_{2}$ can be solved by the method of characteristics. It is easy to get the general solution of Eq. (26) with the form

$$
\varphi_{2}=(C+k r)^{1 / 2} F(\xi),
$$


where

$$
\xi=\int \frac{r \mathrm{~d} \rho}{C+k r}+t
$$

and $F(\xi)$ is a differentiable function of $\xi$. Substituting Eq. (27) into Eq. (25) gives

$$
\varphi_{1}=(C+k r)^{-1 / 2}\left[F^{\prime}(\xi)+(A+k \eta) F(\xi)\right] .
$$

Substituting Eqs. (29) and (27) into Eq. (4), we have

$$
F^{\prime \prime}(\xi)-\beta F(\xi)=0,
$$

where

$$
\beta=(A+k \eta)^{2}+(B+k q)(C+k r) .
$$

By complicated calculations, we can get

$$
\begin{aligned}
& r_{n}=2 \eta \operatorname{sech} \gamma_{n}, \\
& C_{n}=2 \eta k_{n} \operatorname{sech} \gamma_{n} \tanh \gamma_{n}, \\
& A_{n}=\eta k_{n}\left(\operatorname{sech}^{2} \gamma_{n}-\tanh ^{2} \gamma_{n}\right) .
\end{aligned}
$$

Now substituting Eqs. (32) into Eq. (31), we have

$$
\beta_{n}=\left(A_{n}+k_{n} \eta\right)^{2}+C_{n}^{2}-k_{n}^{2} r_{n}^{2}=0 .
$$

Therefore, Eq. (30) is easily solved as

$$
F\left(\xi_{n}\right)=c_{3} \xi_{n}+c_{4},
$$

where $c_{3}$ and $c_{4}$ are the integration constants. Then we obtain the corresponding wave functions:

$\Gamma_{n}=\frac{\operatorname{sech} \gamma_{n}}{1+\tanh \gamma_{\mathbf{n}}}+\frac{1}{2 \eta k_{n} \operatorname{sech} \gamma_{n}\left(1+\tanh \gamma_{n}\right)\left(\xi_{n}+c\right)}$,

$c=\frac{c_{4}}{c_{3}}, \gamma_{n}=2 \eta \rho_{n}+\ln c_{0}$.

Finally, substituting expressions (35) and (32) into Eq. (18), we obtain a family of new solutions for the sine-Gordon equation

$$
\begin{aligned}
u_{n}(x, t)= & n \pi+4 \arctan \left[c_{0} \exp \left(2 \eta \rho_{n}\right)\right]+4 \arctan \Gamma_{n} \\
& n=0, \pm 1, \pm 2, \cdots
\end{aligned}
$$

Next, we investigate this family of new solutions (36). By the transformation (12) and an operation, we rewrite solutions (36) in the coordinates $(X, T)$ as

$$
\begin{aligned}
U_{n}(X, T) & =n \pi+4 \arctan \left[\exp \left(\widetilde{\gamma}_{n}\right)\right]+4 \arctan \left(\widetilde{\Gamma}_{n}\right) \\
n & =0, \pm 1, \pm 2, \cdots,
\end{aligned}
$$

where

$$
\begin{aligned}
\widetilde{\gamma}=\eta\left(1-k_{n}\right)\left(X+\frac{1+k_{n}}{1-k_{n}} T\right)+\ln c_{0}, k_{n}=\frac{(-1)^{n-1}}{4 \eta^{2}} \\
\widetilde{\Gamma}_{n}=\left[\eta(-1)^{n-1} \exp \left(2 \widetilde{\gamma}_{n}\right)+2 \eta(-1)^{n-1} \widetilde{\gamma}_{n}+X-T\right. \\
\left.+2 c+2 \eta(-1)^{n-1}\right]\left\{\left[2 \eta(-1)^{n-1} \widetilde{\gamma}_{n}+X-T+2 c\right]\right. \\
\left.\cdot \exp \left(\widetilde{\gamma}_{n}\right)-\eta(-1)^{n-1} \exp \left(-\widetilde{\gamma}_{n}\right)\right\}^{-1}
\end{aligned}
$$

Then we find

$$
\begin{aligned}
\lim _{x \rightarrow+\infty} U_{n}(X, T)= & \lim _{x \rightarrow-\infty} U_{n}(X, T)=n \pi, \\
& \text { for } \quad|\eta|<\frac{1}{2} \text { and } \eta \neq 0, \\
\lim _{x \rightarrow+\infty} U_{n}(X, T)= & n \pi+4 \pi, \lim _{x \rightarrow-\infty} U_{n}(X, T)=n \pi, \\
& \text { for } \eta>\frac{1}{2}, \\
\lim _{x \rightarrow+\infty} U_{n}(X, T)= & n \pi, \lim _{x \rightarrow-\infty} U_{n}(X, T)=n \pi+4 \pi, \\
& \text { for } \eta<-\frac{1}{2} .
\end{aligned}
$$

So, the solutions (37) are a family of soliton solutions, kink solutions and anti-kink solutions of the sine-Gordon equation, respectively, when $|\eta|<1 / 2$ with $\eta \neq 0, n>1 / 2$ and $\eta<-1 / 2$. In particular, by taking limitations we find that the soliton solutions seemly consist of kinks and anti-kinks pairs bound together. In addition, from Eqs. (38) and (39), the solutions $U_{n}(X, T)$ are functions of $(X-T)$ and $\left(X+T\left(1+k_{n}\right) /\left(1-k_{n}\right)\right)$, thus this family of new solutions contain two traveling-waves with different speeds.

Finally, let $n=0, c=0$ and $c_{0}=1$, we stimulate numerically the solutions $U_{n}(X, T)$ in Figs. 1(a)-1(c), respectively.

From these figures, we can easily find that there exist some oscillations and singularities for the solutions within the local region of space, and we think the occurrence of these phenomena just results from the interaction between two traveling-waves. Therefore, although these solutions are the same as usual soliton, kink and anti-kink solutions from the spatial limitations, there exist obviously large differences in the local region. The further study of these solutions will emerge in the later publication.

Remark: The real parameter $\eta$ just is the imaginary part of one purely imaginary spectrum of the sine-Gordon equation.

\section{REFERENCES}

1 M. C. Cross and P. C. Hohenberg, Rev. Modern Phys. 65 (1993) 851.

2 P. J. Caudrey, J. D. Gibbon, J. C. Eilbech and R. K. Bullough, Phys. Rev. Lett. 30 (1973) 237.

3 R. Hiroto, J. Phys. Soc. Jap. 33 (1972) 1459.

4 A. R. Bishop et al., SIAM J. Math. Anal. 21 (1990) 1511.

5 A. R. Bishop et al., Physica, D 23 (1986) 1.

6 P. S. Lomdahl, O. H. Olsen and M. R. Samuelsen, Phys. Rev. A 29 (1984) 350.

7 E. A. Overman II, A. R. Bishop and D. W. Mclaughlin, Physica, D 19 (1986) 1.

8 YAN Jiaren, TANG Yi and CHEN Zhenhua, Chin. Phys. Lett. 14 (1997) 671.

9 LIU Zengrong and HUANG Debin, Chin. Phys. Lett. 16 (1999) 313.

10 K. Konno and M. Wadati, Progr. Theor. Phys. 53 (1975) 419.

11 M. J. Ablowitz, D. J. Kaup, A. C. Newell and H. Segur, Phys. Rev. Lett. 31 (1973) 125. 